\title{
The LHCb VELO Upgrade
}

\author{
Stefano de Capua ${ }^{* \dagger}$ \\ The University of Manchester $(G B)$ \\ E-mail: stefano.decapua@manchester.ac.uk
}

The upgrade of the LHCb experiment, scheduled for LHC Run-3, will transform the experiment to a triggerless system reading out the full detector at $40 \mathrm{MHz}$ event rate. All data reduction algorithms will be executed in a high-level software farm, enabling the detector to run at luminosities of $2 \times 10^{33} \mathrm{~cm}^{-2} \mathrm{~s}^{-1}$. The Vertex Locator (VELO) is the silicon vertex detector surrounding the interaction region. The current strip detector will be replaced with a hybrid pixel system equipped with electronics capable of reading out at $40 \mathrm{MHz}$. The upgraded VELO will allow for fast pattern recognition and track reconstruction in the software trigger. The silicon pixel sensors have $55 \times 55 \mu \mathrm{m}^{2}$ pitch, and are read out by the VeloPix ASIC. The VeloPix builds on the currently available Timepix3, modified to deliver a radiation hard design capable of an order of magnitude increase in output rate. The hottest regions will have pixel hit rates of 900 Mhits/s, yielding a total data rate more than $3 \mathrm{Tbit} / \mathrm{s}$ for the upgraded VELO. The silicon pixel sensors must be radiation hard to a level of $8 \times 10^{15} 1 \mathrm{MeV} \mathrm{n}_{\mathrm{eq}} \mathrm{cm}^{-2}$, delivered non uniformly over the sensor surface. The R\&D focusses on designs capable of tolerating high voltage after irradiation and maintaining good efficiency and resolution. The detector modules are located in a secondary vacuum, separated from the beam vacuum by a thin custom made foil. The material budget will be minimised by the use of evaporative $\mathrm{CO}_{2}$ coolant, circulating in microchannels within $400 \mu \mathrm{m}$ thick silicon substrates. The current status of the VELO upgrade is described and latest results from the design and prototyping are presented.

38th International Conference on High Energy Physics 3-10 August 2016

Chicago, USA

\footnotetext{
* Speaker.

$\dagger$ on behalf of the LHCb Collaboration
} 


\section{Introduction}

LHCb is a dedicated experiment for the study of flavour physics at the Large Hadron Collider (LHC) at CERN [1]. The experiment is designed to search for evidence of New Physics processes by studying $\mathrm{CP}$ violation and rare decays of heavy flavour mesons. The detector consists of a vertex detector (VELO), dipole magnet, tracking stations, calorimeters and muon stations. The $\mathrm{LHCb}$ detector provides an excellent particle identification, a good momentum resolution and a versatile trigger. During Run-1 and Run-2 of the LHC, the experiment has been running successfully at a maximum instantaneous luminosity of $4 \times 10^{32} \mathrm{~cm}^{-2} \mathrm{~s}^{-1}$, which corresponds to an average rate of 1.5 visible interactions per bunch crossing. The instantaneous luminosity is limited by the trigger, which consists of both hardware and software levels, wth the former limited to a readout of $1 \mathrm{MHz}$ [2]. During Run-3, scheduled to begin in 2021, LHCb plans to operate at a 5 times higher instantaneous luminosity, in order to further improve its physics performance [3]. In order to achieve this, during Long Shutdown 2 (LS2) of the LHC (in 2019 and 2020), LHCb will undergo a major upgrade: the trigger will be redesigned to be purely software and all sub-detectors will be read out at the bunch crossing frequency of $40 \mathrm{MHz}$, which corresponds to an average rate up to 7.2 interactions per bunch crossing [4]. The new trigger design will require the front end electronics to be fully replaced and part of the LHCb detector to be upgraded in order to cope with the increased luminosity. The layout of the upgraded LHCb detector is shown in Figure 1.

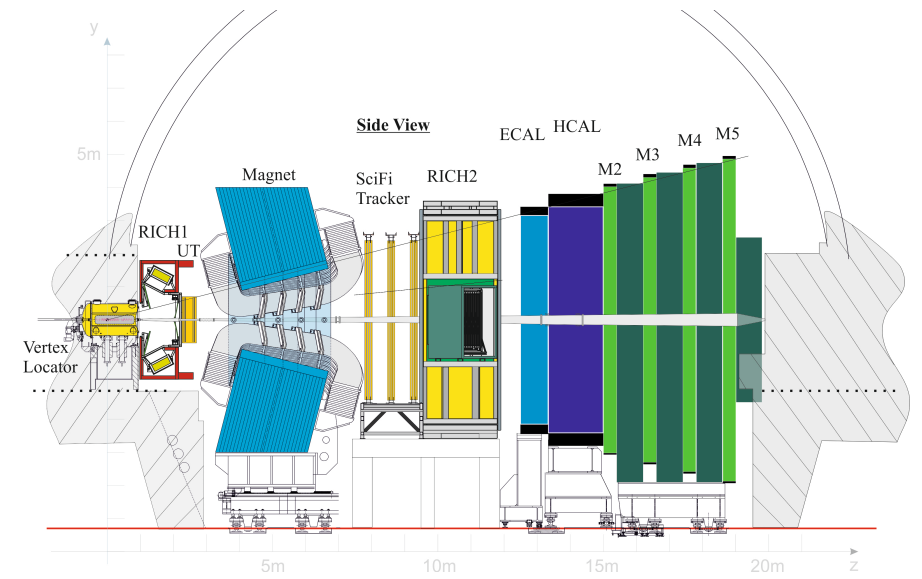

Figure 1: Schematic side view of the upgraded $\mathrm{LHCb}$ detector.

\section{The present Vertex Locator}

The Vertex Locator (VELO) is positioned around the interaction point and ensures precise reconstruction of displaced vertices from the decays of beauty and charm particles, enabling the measurement of hadron lifetimes (of order of $10^{-12} \mathrm{~s}$ ), impact parameters (of $\sim \mathrm{mm}$ ) and flight distances (of $\sim 1 \mathrm{~cm}$ ) [5]. The present VELO consists of two retractable halves, which are retracted by $30 \mathrm{~mm}$ during beam injection and moved into their final position around the luminous region in stable beam conditions, with the closest active strip at $8.2 \mathrm{~mm}$ from the centre. Each half contains 21 double-sided silicon strip detectors, providing measurements of both the radial (R) 
and azimuthal $(\Phi)$ coordinate. The detector is operated in a secondary vacuum tank, separated from the LHC vacuum by a $300 \mu \mathrm{m}$ thick aluminum foil. The sensors are cooled down by an evaporative system using bi-phase $\mathrm{CO}_{2}$ at $-30{ }^{\circ} \mathrm{C}$ (sensors at $-8{ }^{\circ} \mathrm{C}$ ) [6].

During LS2, the present VELO will be replaced by a hybrid pixel detector, with planar silicon sensors. The status of the VELO-Upgrade project is discussed in the following section.

\section{The upgraded Vertex Locator}

The main challenge for the upgraded VELO detector will be to operate at the bunch crossing frequency of $40 \mathrm{MHz}$ and at an instantaneous luminosity of $2 \times 10^{33} \mathrm{~cm}^{-2} \mathrm{~s}^{-1}$. In addition, the detector will also be brought closer to the beams in order to improve the impact parameter resolution, which represents a key performance parameter for selecting decays of beauty and charm hadrons. This can be obtained by reducing the radius at which the first point of the track is measured and the material budget between the primary vertex and the second measured point. Therefore, it is highly desirable to bring the detector as close as possible to the beams and to minimise the material budget. For the upgraded detector, the active area starts at $5.1 \mathrm{~mm}$ from the interaction region.

The upgraded VELO will consist of two halves, each equipped with 26 modules featuring a L-shaped active area of $\sim 24 \mathrm{~cm}^{2}$, placed perpendicular to the direction of the beams, as illustrated in Figure 2 (left). The active area will consist of a $200 \mu \mathrm{m}$ thick n-on-p silicon sensor, bump bonded to three custom made VeloPix ASICs [7]. The VeloPix, based on the Timepix3 ASIC [8], is a binary pixel readout chip with a data driven readout, designed in $130 \mathrm{~nm}$ CMOS technology. It consists of a matrix of $256 \times 256$ pixels, with a pixel pitch of $55 \mu \mathrm{m}$ and capable of withstand very high data rates (up to $900 \mathrm{Mhits} / \mathrm{s}$ ) with a resulting output bandwidth of $16 \mathrm{Gbit} / \mathrm{s}$.

The silicon sensors will be mounted on a $400 \mu \mathrm{m}$ thick silicon substrate, which serves as both mechanical support and thermal interface to the active part of the detector. The main advantage of this solution is that the detector will be composed of silicon parts only and therefore the CTE mismatch can be kept minimal. In addition, the sensors will overhang the substrate by $5 \mathrm{~mm}$ in the inner edge of the module, such that particles will traverse less material in the region near the interaction point and therefore multiple scattering, before the first measuring point, will be reduced.
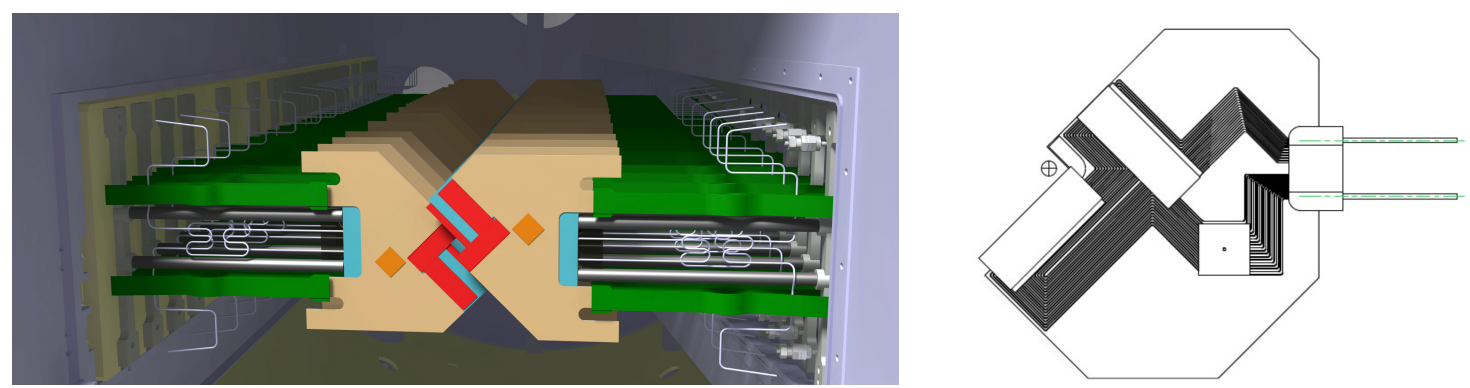

Figure 2: Left: an illustration of the upgraded VELO; silicon sensors are shown in red, silicon substrates in blue. Right: a schematic drawing of the silicon substrate and the incorporated microchannels. 


\subsection{Cooling}

The upgraded VELO will use evaporative $\mathrm{CO}_{2}$ cooling in order to maintain all parts of the detector below $-20{ }^{\circ} \mathrm{C}$ and prevent thermal runaway of the sensor assemblies. In order to fulfil this requirement and remove the expected heat load generated by the electronics mounted on the substrate $(\sim 36 \mathrm{~W})$, the novel microchannel cooling technique [9] has been chosen as baseline solution. This solution consists of nineteen $120 \times 200 \mu \mathrm{m}^{2}$ trenches (microchannels) etched in a $260 \mu \mathrm{m}$ thick wafer, which is then bonded to a cover silicon wafer, thus resulting in the final $400 \mu \mathrm{m}$ thick silicon substrate. Liquid $\mathrm{CO}_{2}$ is injected in the system through a manifold soldered onto the silicon substrate. It then passes through high impedance $60 \times 60 \mu \mathrm{m}^{2}$ restrictions, which have the purpose of triggering the $\mathrm{CO}_{2}$ evaporation. The microchannels are etched following a pattern such that the coolant is let flow directly underneath the components which produce the heat, allowing for a cooling power of more than $36 \mathrm{~W}$ [10]. A schematic view of the microchannel substrate is illustated in Figure 2 (right).

\subsection{RF-box}

As for the current VELO, the upgraded modules will be operated in vacuum and accommodated in a aluminium box, which will separate the VELO volume from the LHC ultra-high vacuum volume. The box also serves as a guide for the mirror currents, shielding the detectors from possible RF pick-up from the beams. The RF-box is therefore required to be vacuum tight, to give a negligible contribution to the impedance of the LHC, to withstand differential pressures of 5 mbar during venting and evacuating procedures, to be thermally stable and radiation hard. Moreover, since the RF-box accounts for most of the material budget of the VELO, it is also required to be made as thin as possible, corrugated and of a high radiation length material. A clearance of only $800 \mu \mathrm{m}$ between the foil and the closest edge of the sensor is also envisaged in order to bring the active area of the detector as close as possible to the beams.

These high demanding requirements make the choice of the material and of the production process particularly challenging. Extensive tests have been carried out to demonstrate the feasibility of constructing a box which meets the requirements, by employing different milling techniques and procedures. A picture of a successfully milled half-length prototype RF-box is shown in Figure 3.

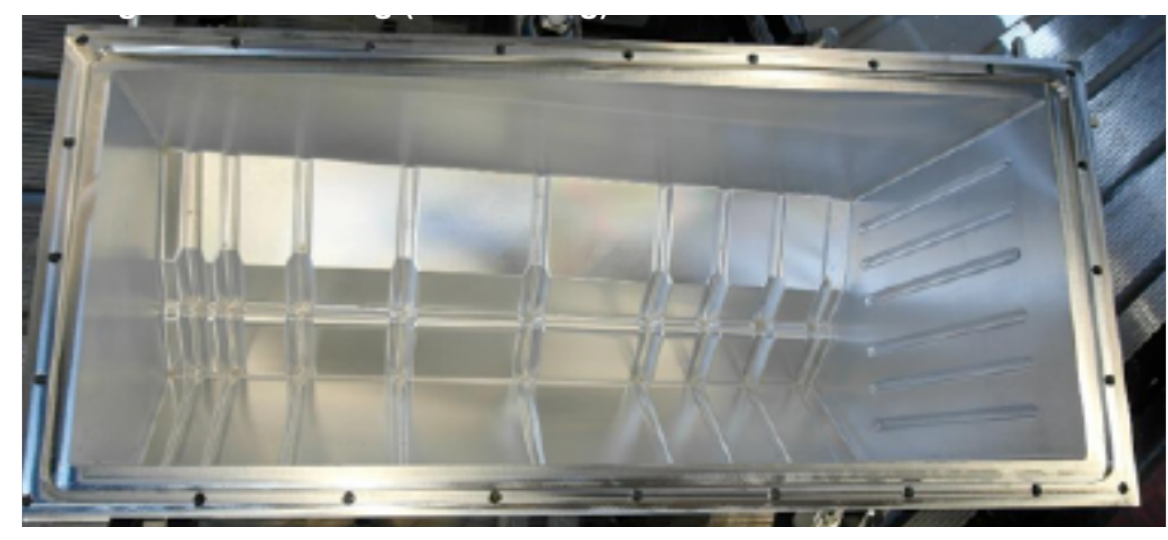

Figure 3: A picture of a half-length prototype RF-box. 


\subsection{Sensors}

The requirements for the upgraded VELO sensors set a very challenging design. The sensors are required to be radiation tolerant in order to prevent a degradation of the performance due to radiation damage. In addition, because of the geometry of the detector, the radiation profile will be highly non uniform: after an integrated luminosity of $50 \mathrm{fb}^{-1}$, the innermost area of the sensors is expected to collect a fluence of $8 \times 10^{15} 1 \mathrm{MeV} \mathrm{n}_{\mathrm{eq}} \mathrm{cm}^{-2}$, while a factor 40 less radiation is expected in the outermost region, which is located at about $40 \mathrm{~mm}$ from the beams. In order to maintain a good signal to noise ratio also after receiving the full fluence, the irradiated sensors are required to collect at least $6000 \mathrm{e}^{-}$per minimum ionising particle with a charge collection efficiency greater than $99 \%$. This can be achieved by applying a high bias voltage ( $1000 \mathrm{~V})$ to the irradiated sensors, but the high dose and the inhomogeneous radiation profile make this very challenging, since the less irradiated regions will have to sustain this high voltage without breaking down.

Since 2014, an extensive test beam campaign has been carried out to qualify a wide range of prototype sensors provided by two manufacturers, Micron and Hamamatsu. Micron provided both n-on-p and n-on-n sensors with a thickness of 200 and $150 \mu$ m respectively, an implant width of $36 \mu \mathrm{m}$ and an inactive edge of 250 or $450 \mu \mathrm{m}$. Hamamatsu provided $200 \mu \mathrm{m}$ thick n-on-p sensors, with an implant width of 35 or $39 \mu \mathrm{m}$ and an inactive edge of 450 or $600 \mu \mathrm{m}$. For testing purposes, all sensors have been bump bonded to TimePix 3 ASICs. Sensors have been irradiated to a fluence of $2-8 \times 10^{15} 1 \mathrm{MeV} \mathrm{n}_{\mathrm{eq}} \mathrm{cm}^{-2}$ in five different facilities, with both uniform (neutrons) and non-uniform (protons) irradiation profiles.

The measurements have shown that, after the full irradiation, the required $6000 \mathrm{e}^{-}$can be achieved below the $1000 \mathrm{~V}$ limit with no breakdown. Results for some of the prototypes are shown in Figure 4. At low voltages, a large drop in efficiency at the edges of the pixel is observed (Fig. 4, left): the effect is due to the distance of the track from the implant, which requires the charge to travel a longer distance. High efficiency is recovered when the bias voltage is increased (Fig. 4, right).

The baseline choice for the upgraded VELO will be $200 \mu \mathrm{m}$ thick n-on-p silicon sensors, bump bonded to three VeloPix chips to form a $14 \times 43 \mathrm{~mm}^{2}$ assembly.
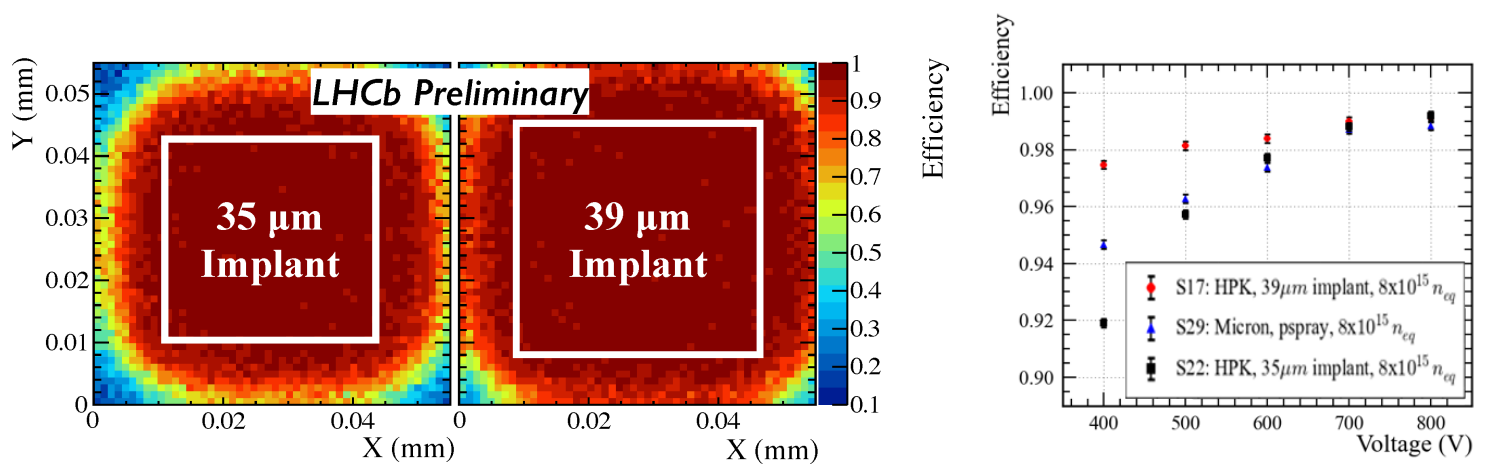

Figure 4: Left: the intra-pixel efficiency for a Hamamatsu sensor at $300 \mathrm{~V}$ with a $35 \mu \mathrm{m}$ implant width (left) and $39 \mu \mathrm{m}$ implant width (right), after being exposed to the full expected fluence. Right: average efficiency versus bias voltage for three irradiated sensors (Hamamatsu and Micron). 


\section{Conclusion}

During the second long shutdown (LS2), the LHCb experiment will be upgraded to sustain a five times higher instantaneous luminosity and collect $50 \mathrm{fb}^{-1}$. In order to achieve this goal, most of the LHCb sub-detectors will be significantly upgraded. The silicon strip detector will be replaced with a hybrid pixel detector, which will be read out at the full $40 \mathrm{MHz}$ bunch crossing rate of the LHC. The active area of the detector will consist of a $200 \mu \mathrm{m}$ thick n-on-p silicon sensor, bump bonded to three custom made VeloPix ASICs. Sensor prototypes have been tested and demonstrated to perform to the required specifications. The silicon sensors will be mounted on a $400 \mu \mathrm{m}$ thick silicon substrate, which will serve as both mechanical support and thermal interface. The project is progressing well in all its areas and is on track for installation in 2020.

\section{References}

[1] The LHCb Collaboration, The LHCb Detector at the LHC, JINST 3 (2008) S08005.

[2] T. Head, The LHCb trigger system, JINST. 9 (2014) no. 09 C09015

[3] The LHCb Collaboration, Letter of Intent for the LHCb Upgrade, CERN-LHCC-2011-001

[4] The LHCb Collaboration, LHCb Trigger and Online Upgrade Technical Design Report, CERN-LHCC-2014-016

[5] The LHCb Collaboration, LHCb VELO Technical Design Report, CERN/LHCC 2001-011, LHCb TDR 5.

[6] B. Verlaat, M. van Beuzekom and A. van Lysebetten, CO-2 cooling for HEP experiments, Published in Naxos 2008, Electronics for particle physics, 328-336, CERN-2008-008.

[7] T. Poikela et al., VeloPix: the pixel ASIC for the LHCb upgrade, JINST 10 (2015) C01057.

[8] T. Poikela et al., Timepix3: a 65K channel hybrid pixel readout chip with simultaneous ToA/ToT and sparse readout, 2014 JINST 9 C05013.

[9] A. Nomerotski et al, Evaporative $\mathrm{CO}_{2}$ cooling using microchannels etched in silicon for the future LHCb vertex detector, JINST 8 (2013) P04004.

[10] O. de Aguiar Francisco et al., Evaporative $\mathrm{CO}_{2}$ microchannel cooling for the LHCb VELO pixel upgrade, JINST 10 (2015) C05014. 